\title{
CAROLINA MICHAËLIS O LOS SABERES DE LA FILOLOGÍA*
}

\author{
JOAQUÍN RUBIO TOVAR \\ Universidad de Alcalá
}

La reciente celebración de un congreso sobre la vida y la obra de Carolina Michaëlis de Vasconcelos (Berlín, 2002) y otro sobre su edición del Cancioneiro da Ajuda (Santiago de Compostela, 2004), muestra el interés que siguen despertando el trabajo y la personalidad de esta filóloga. Uno y otra fueron singulares, pues se desarrollaron en lenguas y culturas tan distintas como la alemana y la portuguesa. Los estudios consagrados a su vida y sus trabajos no han cesado, además, de destacar su condición de mujer, ya que añade para muchos una dimensión que trasciende la aportación de su obra al campo de la filología.

Michaëlis nació en Berlín, «la metrópoli de la inteligencia» como ella solía llamarla, el 15 de marzo de 1851 . Como otras mujeres nacidas en el tardío romanticismo, debió seguir sus estudios universitarios con tutores particulares, mediante la relación epistolar (Gelehrtenkorrespondenz) y, como dice Malkiel, una «intensive reading». En el ambiente en el que se formó Michaëlis no era extraño que las mujeres fueran políglotas, pero no era tan corriente que se consagraran a las lenguas muertas, dedicación esta reservada a los varones. Carolina Michaëlis hablaba varias lenguas vivas y dominaba varias muertas, con lo que participaba de los conocimientos e intereses de hombres y mujeres.

Además de estudiar lenguas, Michaëlis se inició en las múltiples competencias que formaban la filología románica, fundamento de toda su obra, para la

\footnotetext{
* Dona Carolina Michaëlis e os estudos de Filologia Portuguesa, Actas del Colóquio Dona Carolina Michaëlis e os estudos filologicos em Portugal no começo do século xx, 18 de octubre de 2002, Ulrike Mühlschlegel (ed.), Biblioteca Luso-Brasileira 22, Frankfurt am Main, IberoAmerikanisches Institut Preussischer Kulturbesitz, 2004.

o Cancioneiro da Ajuda cen anos despois. Actas do Congreso realizado pola Dirección Xeral de Promoción Cultural en Santiago de Compostela e na lla de San Simón os días 25-28 de maio de 2004, coordinadora científica Mercedes Brea, Santiago de Compostela, Xunta de Galicia, 2004.
}

RFE, LXXXV, 2005, $1 .^{\circ}$, págs. 165-173 
que se había creado un clima apropiado en Berlín. Añádanse a todo ello un talento extraordinario y una capacidad de trabajo fuera de lo común, condiciones que le permitieron escribir tantos y tan importantes estudios. A los catorce años, y tras leer el Nuevo Testamento en español y cotejarlo con el alemán, fue capaz de esbozar una comparación entre ambas lenguas. Guiada por algunos arabistas que frecuentaban la casa paterna, fue ahondando, además, en sus conocimientos de lenguas y literaturas eslavas y semíticas. En estos años de formación es esencial la figura de Karl Goldbeck (1830-1900), que no ha pasado a la historia de la filología como un gran investigador, pero sí como un gran profesor (nada menos). Su conocimiento del español y el portugués, la etimología francesa y el inglés antiguo suscitaron la curiosidad de su alumna. Mérito suyo fue también difundir en Alemania la obra de Antero de Quental. A esta primera formación ella fue añadiendo intereses, como el folclore o el conocimiento de la literatura medieval y renacentista. Michaëlis abordo uno por uno los varios campos de la filología, a saber, la edición de textos, la etimología, la gramática histórica y la historia literaria. Ya casada, en Portugal, acompañaba a su marido en sus investigaciones arqueológicas a distritos rurales y aldeas diminutas y se ocupaba en ejercicios típicos del Wörter und Sachen.

Entre los años de 1867 y 1876 trabajó como traductora oficial de portugués y español, pero no abandonó su quehacer filológico. Durante este período publicó una serie de ediciones de textos españoles y portugueses (Tres flores del teatro antiguo español, en 1870; Romancero del Cid, en 1871; Fiori della poesia italiana antica e moderna, también en 1871 y Os Lusíadas de Camōes, de 1873). En 1876 apareció su primer trabajo de historia de la lengua, Studien zur romanischen Wortschöpfung, que fue alabado por Meyer-Lübke en el célebre artículo que dedicó a la autora en 1927. Para el maestro alemán, era infrecuente encontrar en una primera investigación un ejemplo de ciencia tan rigurosa, fundamentada en datos sólidos, contrastados, y no en especulaciones vagorosas. Estos fueron sus primeros trabajos, anteriores todos a 1876, año en el que contrajo matrimonio con Joaquim de Vasconcelos, que estaba llamado a ser uno de los fundadores de la historia del arte en Portugal.

Su compromiso con la cultura y la sociedad portuguesas, siendo como era alemana de cuna, otorga a su personalidad una dimensión muy peculiar. Muchos estudios dedicados a la obra de Michaëlis se refieren en algún momento a su condición de «intermediaria entre la cultura neolatina y la alemana», de acuerdo con la doble nacionalidad a la que tantas veces se refirió ella. La investigadora dio a conocer a los portugueses una parte de su pasado, al mostrarles documentos inéditos de su historia, y presentarlos dentro de la cultura europea. Y, por otro lado, ilustró a los lectores cultos alemanes sobre la grandeza 
del pasado portugués. Michaëlis escribió en alemán una Geschichte der portugiesischen Literatur, que aparece en el volumen segundo del Grundriss de Gröber. Además de esta contribución, no debe olvidarse que las Poesías de Francisco de Sá Miranda y los dos volúmenes del Cancioneiro da Ajuda vieron la luz en Alemania. Su Prática de três pastores na noite de Natal apareció en la editorial Braunschweig en 1881 bajo el título de Ein Portugiesisches Weihnachtsauto: Prática de três Pastores, que ella dedicó a la Berliner Gesellschaft für das Studium der Neuren Sprachen a la que pertenecía en calidad de socia honoraria desde 1877.

La vida en Portugal la llevó a comprometerse con aquella realidad, tan distinta a la alemana. No era, desde luego, la primera vez que se había interesado por el retraso cultural portugués, pues había terciado en una célebre polémica acerca de la poco rigurosa traducción del Fausto de Goethe llevada a cabo por António Feliciano de Castilho y que fue denunciada por Vasconcelos, Téfilo Braga y Adolfo Coelho entre otros. Carolina Michaëlis se hico eco de la polémica en un artículo publicado en Magazin für die Literatur des Auslandes en 1873. Los tres investigadores citados enarbolaron el estandarte de los ímpetus regeneracionistas en Portugal, y editaron, bajo la dirección de Coelho, una revista titulada Bibliografia Crítica de Historia e Literatura. Allí apareció una crítica muy elogiosa de Téffilo Braga a la edición del Romancero del Cid de Michaëlis y leeremos también una favorable reseña de la propia Carolina al Dictionnaire d'Etymologie Française de August Scheler. Poco a poco fueron apareciendo estudios sobre la literatura medieval y renacentista portuguesa. Son trabajos que se enmarcan en el campo de la filología, que ella misma definió como: «o estudo científico, histórico e comparado da lengua nacional em toda sua amplitude, nāo só quanto à gramática (fonética, morfologia, sintaxe) e quanto à etimologia, semasiologia, etc., mas também como órgao da literatura e como manifestação do espírito nacional»"

Esta definición, esta visión tan amplia se corresponde con sus estudios dedicados a aspectos etimológicos, semánticos, ortográficos, morfológicos, sintácticos, literarios, folklóricos, históricos, biográficos y etnográficos ${ }^{2}$. Añádanse las ediciones críticas de las poesías de Francisco de Sá de Miranda (1885), del Cancioneiro da Ajuda (1904), las Randglossen zum altportugiesischen Liderbuch, publicadas en la ZRPH que editaba Gröber, y los Estudos sobre o Romanceiro peninsular, romances velhos em Portugal (1907-1909) escritos a petición de Menéndez Pidal o las excepcionales Notas vicentinas (entre 1912 y

\footnotetext{
1 Liçoes de Filologia Portuguesa segundo as preleccoes feitas aos cursos de 1911/12 e de 1912/13, ediçao da Revista de Portugal, Lisboa. 1946, 152.

2 Aparecen muchos de ellos en Dispersos. Originais portugueses (I Varia, II Lingüística, II Camoniana), Lisboa, Ediçao de Ocidente e Revista de Portugal, 1964.
} 
1922) que iban a servir de notas preliminares a una edición crítica de las obras de Gil Vicente. De 1920 son sus «Miscelas etimológicas». Un sugestivo estudio titulado A saudade portuguesa (1914), que nace de una antigua canción portuguesa inserta en un drama castellano sobre doña Inés de Castro, le sirvió para escribir «divagaçoes filológicas, ligeiramente retintas de filosofia». Esta orientación no es extraña a los saberes de la filología y movió la pluma de numerosos investigadores. No debe olvidarse, por ejemplo, que para Leite de Vasconcelos, la História da lingoa portuguesa que proyectaba escribir debía culminarse con un capítulo sobre «A língoa como expressão da alma e vida do povo português».

He mencionado al principio de estas líneas que los estudiosos de la obra de nuestra filóloga no han dejado de destacar su condición de mujer, hecho este que no es de extrañar. En primer lugar, Michaëlis reconocía lo excepcional de su caso. Aunque sus trabajos fueran elogiados por Meyer Lübke y reconocidos por G. I. Ascoli, G. Paris o A: Mussafia, lo cierto es que supo de ciertas ironías y malos modos de más de un filólogo. Michaëlis sabía que sus conocimientos y su obra estaban a la altura (y por encima de) los trabajos de muchos colegas varones, de ahí que en más de una ocasión se viera obligada a reaccionar ante la postergación a la que algunos quisieron condenarla ${ }^{3}$. Ernesto Monaci pretendió reducir en una publicación colectiva la aportación de Michaëlis al Cancioneiro da Ajuda a un mero apéndice, eliminando muchos de los textos en los que ella había trabajado durante años. Tavani recordaba la reacción de la filóloga en su brillante trabajo:

Carolina Michaëlis era persoa demasiado educada e intelixente, e tamén demasiado consciente, por experiencia propia, da subalternidade feminina nun mundo organizado polos e para homes, para reaccionar directamente á falta de tacto de Monaci, cuxas intencións eran manifestamente limitativas da liberdade intelectual dunha investigadora estrangeira que nin tan só era alumna súa ${ }^{4}$.

Por otro lado, nuestra autora sabía bien de la miserable postergación que sufrían las mujeres en Portugal. Michaëlis denunció la falta de formación que padecían y la necesidad de que tuvieran acceso al saber. En 1896 se hizo eco de un congreso feminista en Berlín con un ensayo que apareció en el diario de $O$ comércio do Porto, y en 1902 aparecio «O movimento feminista em Portugal» en el diario $O$ Primeiro de Janeiro.

\footnotetext{
${ }^{3}$ En la Freie Universität de Berlin se está desarrollando un proyecto sobre fa aportación de la mujeres al campo de la romanística. Sus líneas de investigación pueden consultarse en bttp:/l www.lingrom.fu-berlin.de/frauen-in-der-romanistik/

${ }^{4}$ G. Tavani, «Carolina Michaëlis e a crítica do texto, cen anos despois da edición de Halle», o Cancioneiro da Ajuda cen anos despois, Xunta de Galicia, 2004, pág. 60.
} 
La profesora Ulrike Muehlschlegel ha reunido en un volumen las ponencias presentadas en el congreso organizado en Berlín en el año de 2002. Estas actas nos presentan algunas perspectivas renovadoras para comprender la figura de Michaëlis. Por un lado, muestran cómo fue la formación de una mujer nacida alemana en el último tercio del siglo XIX, es decir, cuáles fueron sus lecturas, los maestros e ideas que la influyeron, las teorías contra las que reaccionó, pero también el modo en que desarrollo su docencia en la universidad portuguesa de la época. Es de enorme interés el trabajo del profesor Thieleman para conocer la metodología que inspiró su Estudio sobre la formación de palabras (1876), las opciones que siguió en el marco del pensamiento lingüístico germano del siglo XIX (Humboldt, Grimm, Diez, Schleicher) y también la influencia del francés Michel Bréal y sus opiniones expresadas en «De la forme et de la fonction des mots». Gabriele Beck-Busse traza en «Carl Goldbeck - 'amigo e mentor'» un retrato del profesor de las hermanas Michaëlis - Henriette y Carolina-, que fue decisivo para su formación de filólogas, al tiempo que presenta un panorama sobre la educación de la mujer y sobre el papel que cumplieron los círculos eruditos en el Berlín del siglo XIX. Por su parte, Ricarda Muser muestra, a través de numerosos documentos, cómo era la educación de las mujeres en la época en la que Carolina Michaëlis desarrolló su investigación. Clarinda de Azevedo describe, gracias a su revisión de documentos del archivo universitario, una imagen detallada y precisa de la época en la que la filóloga enseñó en la universidad de Coimbra, y José Augusto Seabra reflexiona sobre doña Carolina y la saudade.

Mercedes Brea es la coordinadora científica del congreso sobre el Cancioneiro da Ajuda, uno de los más importantes manuscritos conservados en las bibliotecas portuguesas. Como es sabido, se trata de un cancionero colectivo que recoge más de trescientos poemas, entre los que no faltan cantigas d'amor al estilo de la cansó trovadoresca, de autores que participaron en las cortes de Portugal y de Castilla a lo largo del siglo XIII.

La importancia de la edición de la filóloga portuguesa y el magnetismo que encierran los poemas recogidos ha convocado a profesores e investigadores de las universidades de Santiago de Compostela, La Coruña, Zurich, California, Santa Bárbara, La Sapienza, Ginebra, Padua, Lisboa, Coimbra, Oporto, Murcia, Vigo, Nantes, París, el Centro Ramón Piñeiro..., lo que da idea de la dimensión internacional del encuentro. En líneas generales puede decirse que el congreso ha permitido recordar cómo planteó doña Carolina su investigación, ha invitado a revisar su propuesta, y a actualizar y superar algunas de sus investigaciones. Son muchas las cuestiones (paleográficas, lingiuísticas, sobre la naturaleza del texto, la cronología de las composiciones recogidas o el origen geográfico de algunas composiciones) que debían revisarse y actualizarse. Las 
contribuciones de las Actas van en esta dirección y es muy de agradecer la oportunidad del congreso organizado por la profesora Brea. La edición de Michaëlis no ha sido superada completamente. Su solidez, al igual que la de los libros de otros sabios filologos de su generación, proviene de haberse realizado sin prisa, comprobando y valorando los datos, tras muchísimas horas de reflexión y análisis. La investigadora trabajaba sin la urgencia que impone la burocracia universitaria que hoy padecemos. Sin embargo, el tiempo no ha pasado en balde y hay una legión de estudiosos que proponen nuevas soluciones y plantean nuevos problemas.

Los primeros trabajos de la filóloga parece que datan de la primavera de 1877 («meses felizes e saudosos (de Maio a setembro de 1877) gastei na empresa de descifrar e copiar, com paixão e paciência, essas páginas seis vezes seculares»), pero la tarea no se vio cumplida hasta 1904. Esto significa que desde el primer encuentro con el manuscrito hasta su publicación transcurrieron veintisiete años. Aunque la filóloga portuguesa alternara esta investigación con otros trabajos y con su actividad como docente (hubo cursos en los que llegó a enseñar cinco materias diferentes: filología románica, filología portuguesa y lengua y literatura alemanas en tres niveles diferentes), lo cierto es que la dedicación mayor fue el Cancioneiro. Maria Ana Ramos, una de las mayores autoridades en este texto, se hacía al principio de su sólido estudio una pregunta fundamental: «A primera interpelação que se nos coloca hoje é justamente como explicar este espaço de tempo decorrido entre 1877 e 1904$)^{5}$. Es una pregunta clave, que convoca otras muchas. Conviene plantearse cuál era la formación de Michaëlis en 1874. La investigadora no podía desconocer las ideas y los trabajos de Karl Lachmann, pues tanto E. Maetzner como Karl Goldbeck le habrían explicado la metodología del gran maestro de la ecdótica. La influencia de Lachmann se había dejado sentir en Alemania (en las ediciones de Carl Appel o de Bartsch), pero no era una línea predominante y existían otros procedimientos como el que proponía Carl Mahn en sus libros Die Werke der Trobadours (1846-1853) y Gedichte der Troubadours (1856-1873), ambos publicados en Berlín. De ambas líneas de investigación tuvo conocimiento $\mathrm{Ca}^{-}$ rolina Michaëlis, pues mantenía correspondencia con investigadores que defendían una u otra hipótesis. Dice Tavani que compartía con ellos: "As experiencias, mais non todas as conviccións: probablemente porque, practicando tradicións textuais de dimensións moi reducidas como as peninsulares, non tiña as oportunidades de aplicar a esas tradicións as novas técnicas reconstructivas utilizadas polos editores da poesía provenzal, francesa ou italiana, que lidaban con decenas de manuscritos e cunha varia lectio de proporcións ás veces

5 Maria Ana Ramos, «O Cancioneiro ideal de D. Carolina», en O Cancioneiro..., 2004, pág. 14. 
asombrosas» ${ }^{6}$. Sin duda, la filóloga portuguesa no siguió al pie de la letra las ideas de Lachmann; es más, tal y como señala Ramos, «C. Michaëlis um pouco avant la lettre dava conta de certa inoperatividade do método de Lachmann» ${ }^{7}$. Los instrumentos, los métodos de la crítica textual que conocía la llevaron a enmendar, a reconstruir aquí y allá, aunque, como dice Ramos: «nem sempre se apreenda o critério a que submeten a emendatio» ${ }^{8}$. Da la sensación de que Michaëlis editaba el texto al tiempo que se preguntaba cómo debía editarse un texto así. En ningún caso hubo una tarea mecánica, sino una indagación permanente para entender los poemas, su métrica, la historia y naturaleza del códice, la necesidad de responder a muchas dudas. Michaëlis «vai justamente diligenciar de modo a que o Cancioneiro da Ajuda, fragmentado, incompleto, possa transformar-se também, enquanto objecto, em um Cancioneiro ideal que tenia contido a produçāo que lhe era materialmente possível reconstituins?. Los estudios de Ramos, Tavani o Mariña Arbor Aldea, entre otros, plantean preguntas y sugieren respuéstas de enorme interés para entender la clase de hipótesis de la que partió Michaëlis y cómo el contacto con los poemas, con la naturaleza del cancionero, le hizo variar criterios, plantearse ante qué clase de texto estaba y cómo quería editarlo.

La convivencia con un texto durante veintisiete años no es sencilla y no va en una dirección única, no es una línea recta que va del filólogo al texto. El filólogo trabaja sobre la obra, pero ésta influye también en él. Michaëlis fue reflexionando sobre la naturaleza del manuscrito que tenía delante, la clase de obra ante la que se encontraba, al tiempo que escrutaba las palabras, los nombres, el sentido de los poemas, la biografía de los trovadores, la métrica de las composiciones, la naturaleza y factura del códice. Tanto trabajo, tanta dedicación, tanta experiencia acumulada no impidieron que escribiera al frente de la edición que:

Se hoje recomeçasse, seguia outro rumo. Há muito que reconheci quanto melhor teria sido dar logo em 1880 a edição paleográfica para fazer corpo com os outros dois Cancioneiros; levar a eito numa Quarta Parte a restituição integral dos textos todos, logo que Ernesto Monaci nos tivesse revelado as variantes do Cancioneiro Colocci Brancuti e o estudo prometido.

Uno no puede dejar de percibir una melancolía parecida a la que invadió el corazón de Gibbon al ver concluir la obra en la que había invertido treinta años de su vida.

\footnotetext{
${ }^{6}$ Tavani, art. cit., pág. 58.

${ }^{7}$ Ramos, art. cit., pág. 19.

${ }^{8}$ Ibídem, pág. 17.

${ }^{9}$ Ibíd, pág. 19.
} 
La meticulosidad del filólogo, el afán por acumular datos, ordenarlos y valorarlos, fueron sus tareas esenciales, y las que le hicieron variar de opinión («quanto maior número de factos apurava, tantos mais problemas surjiam»). En una carta a Alfredo Pimenta del 7 de diciembre de 1905 confesaba que padecía de micr - opia, de afán por los datos, por controlar la más pequeña observación. Esta manera de trabajar tan rigurosa ha sido la que ha permitido a los filologos construir obras perdurables y caracteriza muy bien el modo de hacer de Michaëlis. En la primera de las Notas vicentinas escribía: «Tentarei averiguar factos, remontando as fontes, sempre que isso me seja possível. [...] Servindo-me de indícios dispersos, ponderando, sem juízo preestabelecido, hipóteses apresentadas já por outrem, sujeitando à critica tradições e lendas, não dando fé a nenhuma, embora seculares às vezes exageradas onde quer que seja e por quem quer que seja, quando nâo forem suficientemente documentadas» ${ }^{10}$. La idea de investigación y de cultura chocan frontalmente con la prisa y el exhibicionismo. Al frente de su estudio sobre A Infanta D. Maria de Portugal (1521-1577) e a suas Damas escribe que: «A verdadeira cultura, excluindo ostentaçôes pedantescas, obriga a uma longa e árdua iniciação»" ${ }^{11}$.

$\mathrm{El}$ contenido y la orientación de los estudios recogidos en las Actas del congreso celebrado en Santiago son muy variados, como no podía ser de otra manera, dada la enorme cantidad de problemas que abordó Carolina Michaëlis en su edición. Además de las cuestiones ecdóticas que he recordado más arriba, los investigadores han vuelto a pensar sobre la métrica y la música de los poemas (Billy, Arbor, Canettieri, Pulsoni, Ferreira, Ripoll Anta) han reflexionado sobre los géneros y temas trovadorescos representados en el Cancioneiro (Frateschi), sobre la autoría de las composiciones e identificación de los poetas (Antonia Vínez, Zapico, Gaiferos), la presencia de temas y motivos en los poemas (el amor y la constelación que rodea el sentimiento, el mar y las aguas, la muerte, el mito de Tristán: Castelucci, Gouiran, Carmona, Vallín, etc), la presencia de textos alfonsíes en la colección (Paredes), la visión que doña Carolina ofrecía sobre la lírica tradicional (Alvar), la presencia del universo gallego en el Cancioneiro (Lorenzo Gradín, Ferreira-Ribeiro, Arias Frexedo, Martínez Pereiro, Couceiro, Pena, Gutiérrez García, Pérez Barcala) etc. El volumen recoge cuarenta artículos de los que resulta imposible dar cuenta en estas líneas.

No debe ser casual, dice con razón Mercedes Brea al frente del volumen, que doña Carolina naciera en Alemania, se iniciara en este país a la romanística y fuera una autoridad en literatura gallego-portuguesa, ni debe ser tampoco fruto del azar que la gran autoridad en esta literatura sea hoy un italiano, el maestro Giuseppe Tavani (italiano fue también Angelo Colocci). La

\footnotetext{
${ }^{10}$ Notas vicentinas, Lisboa, 1949, pág. 1.

"I Porto, 1902, pág. 74.
} 
indicación de la profesora Brea invita a una reflexión. La formación de Michaëlis hunde sus raíces en la filología románica. El lugar de nacimiento y formación le permitían leer alemán, latín, español, italiano, catalán, portugués y algunas lenguas eslavas y semíticas. Sobra decir que su formación y curiosidad le permitían moverse por otras lenguas románicas con enorme facilidad. Así las cosas, conviene recordar el absurdo que supone pensar en un filologo encerrado en una única lengua y despreciar la formación que aportaba la filología románica (me he permitido llamarla La vieja diosa en un reciente ensayo) ${ }^{12}$. La metodología y la orientación de aquellas investigaciones ha quedado arrinconada en los planes de estudios de muchas universidades europeas, $y$ hay que lamentar esta postergación, porque son muchas las investigaciones que no podrán realizarse si se ignora el caudal de saberes y la metodología que bullían en el núcleo de la vieja filología.

Cabe hacer, para terminar, alguna reflexión acerca de la obra de doña Carolina en estos tiempos en los que la prisa y la urgencia de resultados impiden realizar los trabajos con el ritmo de antaño. Tras cien años del Cancioneiro de Ajuda, los especialistas se reúnen para decir, con razón, que hoy las cosas no pueden ni deben plantearse como lo hizo doña Carolina, y que sabemos más que ella. Se ha recomendado, y así debe ser, preparar una edición digitalizada que permita acceder a la vieja edición de Michaëlis y mantener al día las nuevas aportaciones de los investigadores ${ }^{13}$. El nuevo formato permitirá una renovación permanente de nuestro conocimiento del Cancioneiro. Los proyectos de investigación, el saber de los nuevos especialistas en cada una de las parcelas que abordó Michaëlis en solitario y los potentes y útiles medios informáticos darán pronto a la luz un texto renovado, que podrá revisarse fácil y rápidamente, y que superará el saber de doña Carolina. No harán falta tantos años como los empleados por la filóloga alemana para llevar a buen puerto esta empresa. Estoy deseando reseñar esta nueva y necesaria edición del Cancioneiro, aunque no creo que esté cien años en cartel.

\footnotetext{
12 Joaquín Rubio Tovar, La vieja diosa. De la Fîlología a la Posmodernidad, Alcalá, Centro de Estudios Cervantinos, 2004.

${ }^{13}$ El profesor Harvey L. Sharrer («Estado actual de los Estudios sobre el Cancioneiro da Ajuda, págs. 41-54) es autor de un artículo clarificador en el que señala a los investigadores algunas de las tareas que deberian emprenderse.
} 